\title{
A novel derivation for modal derivatives based on Volterra series representation and its use in nonlinear model order reduction
}

Maria Cruz Varona ${ }^{1}$, Raphael Gebhart ${ }^{1}$, Philip Bilfinger ${ }^{1}$, Boris Lohmann ${ }^{1}$, Daniel J. Rixen ${ }^{2}$

${ }^{1}$ Chair of Automatic Control, Technical University of Munich

${ }^{2}$ Chair of Applied Mechanics, Technical University of Munich

COMPDYN-UNCECOMP 2019

MS 27: Advances in MOR techniques for CSD

Crete, June 26th 2019

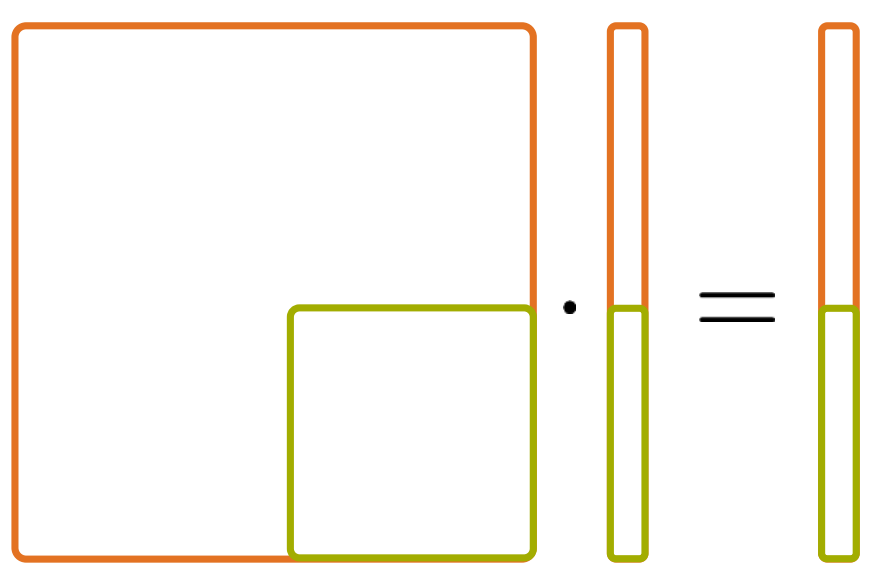




\section{Projective Model Order Reduction}

Nonlinear second-order full order model (FOM)

$$
\begin{aligned}
\boldsymbol{M} \ddot{\boldsymbol{q}}(t)+\boldsymbol{D} \dot{\boldsymbol{q}}(t)+\boldsymbol{f}(\boldsymbol{q}(t)) & =\boldsymbol{B \boldsymbol { F } ( t )} \quad \boldsymbol{q}(0)=\boldsymbol{q}_{0}, \dot{\boldsymbol{q}}(0)=\dot{\boldsymbol{q}}_{0} \\
\boldsymbol{y}(t) & =\boldsymbol{C \boldsymbol { q } ( t )}
\end{aligned}
$$

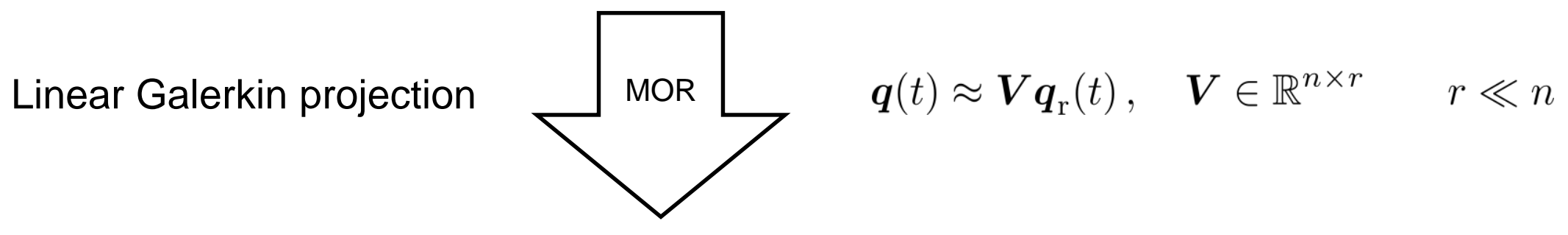

Reduced order model (ROM)

$$
\begin{aligned}
\boldsymbol{M}_{\mathrm{r}} \ddot{\boldsymbol{q}}_{\mathrm{r}}(t)+\boldsymbol{D}_{\mathrm{r}} \dot{\boldsymbol{q}}_{\mathrm{r}}(t)+\boldsymbol{V}^{\top} \boldsymbol{f}\left(\boldsymbol{V} \boldsymbol{q}_{\mathrm{r}}(t)\right) & =\boldsymbol{B}_{\mathrm{r}} \boldsymbol{F}(t) \quad\left\{\boldsymbol{q}_{\mathrm{r}}(0), \dot{\boldsymbol{q}}_{\mathrm{r}}(0)\right\}=\left(\boldsymbol{V}^{\top} \boldsymbol{V}\right)^{-1} \boldsymbol{V}^{\top}\left\{\boldsymbol{q}_{0}, \dot{\boldsymbol{q}}_{0}\right\} \\
\boldsymbol{y}_{\mathrm{r}}(t) & =\boldsymbol{C}_{\mathrm{r}} \boldsymbol{q}_{\mathrm{r}}(t)
\end{aligned}
$$

with

$$
\begin{aligned}
& \left\{\boldsymbol{M}_{\mathrm{r}}, \boldsymbol{D}_{\mathrm{r}}\right\}=\boldsymbol{V}^{\top}\{\boldsymbol{M}, \boldsymbol{D}\} \boldsymbol{V}, \quad \boldsymbol{f}_{\mathrm{r}}(\boldsymbol{q})=\boldsymbol{V}^{\top} \boldsymbol{f}\left(\boldsymbol{V} \boldsymbol{q}_{\mathrm{r}}\right) \quad \text { Hyper-reduction! } \\
& \boldsymbol{B}_{\mathrm{r}}=\boldsymbol{V}^{\top} \boldsymbol{B} \\
& C_{\mathrm{r}}=\boldsymbol{C} \boldsymbol{V}
\end{aligned}
$$




\section{Nonlinear dimensional reduction methods}

\section{Simulation-based approaches (e.g. POD)}

Take snapshots of the simulated trajectory for typical (training) input force and perform SVD

$$
\begin{array}{lr}
\underset{\left(n, n_{\mathrm{s}}\right)}{\boldsymbol{Q}}=\left[\boldsymbol{q}\left(t_{1}\right), \boldsymbol{q}\left(t_{2}\right), \cdots, \boldsymbol{q}\left(t_{n_{\mathrm{s}}}\right)\right] & \text { Reduction basis: } \boldsymbol{V}=\boldsymbol{M}_{\mathrm{r}} \in \mathbb{R}^{n \times r} \\
\boldsymbol{Q} \stackrel{\mathrm{SVD}}{=} \underset{(n, n)}{\boldsymbol{M}} \underset{\left(n, n_{\mathrm{s}}\right)}{\boldsymbol{\Sigma}} \underset{\left(n_{\mathrm{s}}, n_{\mathrm{s}}\right)}{\boldsymbol{N}^{\top}} \approx \underset{(n, r)\left(r, n_{\mathrm{s}}\right)}{\boldsymbol{M}_{\mathrm{r}} \underset{\left(n_{\mathrm{s}}, n_{\mathrm{s}}\right)}{\boldsymbol{\Sigma}_{\mathrm{r}}} \underset{\boldsymbol{N}^{\top}}{\boldsymbol{q}}} & \boldsymbol{q}(t) \approx \boldsymbol{V} \boldsymbol{q}_{\mathrm{r}}(t)=\sum_{i=1}^{r} \boldsymbol{v}_{i} q_{\mathrm{r}, i}(t)
\end{array}
$$

\section{Simulation-free / System-theoretic methods}

- Basis augmentation: Enrichment of a linear basis with nonlinear information

$$
\boldsymbol{V}_{\text {aug }}=\left[\boldsymbol{V}^{(1)}, \boldsymbol{V}^{(2)}\right] \quad \boldsymbol{q}(t) \approx \boldsymbol{V}_{\text {aug }} \boldsymbol{q}_{\mathrm{r}, \text { aug }}(t) \quad \begin{aligned}
& + \text { : Easy projection } \\
& -: \text { Higher reduced order }
\end{aligned}
$$

- Nonlinear projection (e.g. Quadratic Manifold)

$$
\begin{aligned}
& \boldsymbol{V}^{(1)} \in \mathbb{R}^{n \times r} \\
& \boldsymbol{V}^{(2)} \in \mathbb{R}^{n \times r^{2}} \\
& \boldsymbol{q}(t) \approx \boldsymbol{V}^{(1)} \boldsymbol{q}_{\mathrm{r}}(t)+\boldsymbol{V}^{(2)}\left(\boldsymbol{q}_{\mathrm{r}}(t) \otimes \boldsymbol{q}_{\mathrm{r}}(t)\right) \quad+: \text { Smaller reduced order } \\
& \text { - : Difficult projection }
\end{aligned}
$$

$$
\text { Reduced coordinates: } \boldsymbol{q}_{\mathrm{r}}(t)=\left[q_{\mathrm{r}, 1}(t), \cdots, q_{\mathrm{r}, r}(t)\right]^{\top}=\left[\eta_{1}(t), \cdots, \eta_{r}(t)\right]^{\top}
$$




\section{Original derivation for modal derivatives}

Idea: Compose reduction basis with both vibration modes and modal derivatives

$$
\boldsymbol{V}_{\text {aug }}=\left[\boldsymbol{\Phi}_{r}, \boldsymbol{\Theta}_{r^{2}}\right] \quad \boldsymbol{\Phi}_{r}=\left[\boldsymbol{\phi}_{1, \mathrm{eq}}, \ldots, \boldsymbol{\phi}_{r, \mathrm{eq}}\right] \in \mathbb{R}^{n \times r} \quad \boldsymbol{\Theta}_{r^{2}}=\left[\boldsymbol{\theta}_{11}, \ldots, \boldsymbol{\theta}_{r r}\right] \in \mathbb{R}^{n \times r^{2}}
$$

1.) Vibration modes of the linearized model:

Linearization point: $\boldsymbol{q}_{\mathrm{eq}}$

$$
\left(\boldsymbol{K}_{\mathrm{eq}}-\omega_{i, \mathrm{eq}}^{2} \boldsymbol{M}\right) \phi_{i, \mathrm{eq}}=\mathbf{0} \quad i=1, \ldots, r
$$

$$
\boldsymbol{K}_{\mathrm{eq}}=\boldsymbol{K}\left(\boldsymbol{q}_{\mathrm{eq}}\right)=\left.\frac{\partial \boldsymbol{f}(\boldsymbol{q}(t))}{\partial \boldsymbol{q}(t)}\right|_{\boldsymbol{q}_{\mathrm{eq}}}
$$

Normalization condition: $\quad \phi_{i, \mathrm{eq}}^{\top} \boldsymbol{M} \phi_{i, \mathrm{eq}}=1$

2.) Perturbation of eigenmodes:

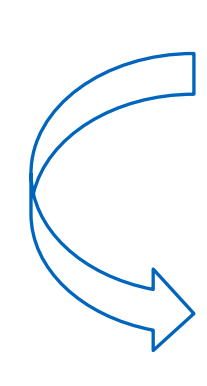

$$
\begin{gathered}
\frac{\partial}{\partial \eta_{j}(t)}\left(\boldsymbol{K}\left(\boldsymbol{q}_{\mathrm{eq}}\right)-\omega_{i}^{2}\left(\boldsymbol{q}_{\mathrm{eq}}\right) \boldsymbol{M}\right) \boldsymbol{\phi}_{i}\left(\boldsymbol{q}_{\mathrm{eq}}\right)=\mathbf{0} \\
\left(\frac{\partial \boldsymbol{K}_{\mathrm{eq}}}{\partial \eta_{j}(t)}-\frac{\partial \omega_{i, \mathrm{eq}}^{2}}{\partial \eta_{j}(t)} \boldsymbol{M}\right) \boldsymbol{\phi}_{i, \mathrm{eq}}+\left(\boldsymbol{K}_{\mathrm{eq}}-\omega_{i, \mathrm{eq}}^{2} \boldsymbol{M}\right) \frac{\partial \boldsymbol{\phi}_{i, \mathrm{eq}}}{\partial \eta_{j}(t)}=\mathbf{0}
\end{gathered}
$$$$
\begin{aligned}
& i=1, \ldots, r \\
& j=1, \ldots, r
\end{aligned}
$$

Modal derivatives (MDs): $\boldsymbol{\theta}_{i j}=\frac{\partial \phi_{i, \mathrm{eq}}}{\partial \eta_{j}(t)}$ 


\section{Modal derivatives}

\section{Calculation formula}

$$
\boldsymbol{\theta}_{i j}=\frac{\partial \phi_{i, \mathrm{eq}}}{\partial \eta_{j}(t)}
$$

$$
\left(\boldsymbol{K}_{\mathrm{eq}}-\omega_{i, \mathrm{eq}}^{2} \boldsymbol{M}\right) \boldsymbol{\theta}_{i j}=\left(\frac{\partial \omega_{i, \mathrm{eq}}^{2}}{\partial \eta_{j}(t)} \boldsymbol{M}-\frac{\partial \boldsymbol{K}_{\mathrm{eq}}}{\partial \eta_{j}(t)}\right) \boldsymbol{\phi}_{i, \mathrm{eq}}
$$$$
\begin{aligned}
& i=1, \ldots, r \\
& j=1, \ldots, r
\end{aligned}
$$

Singular linear system of equations

$$
\mathbf{A} \mathbf{x}=\mathbf{b}
$$

\section{Right-hand side (rhs)}

- Derivative of eigenfrequencies: $\quad \boldsymbol{\phi}_{i, \mathrm{eq}}^{\top} \boldsymbol{M} \boldsymbol{\phi}_{i, \mathrm{eq}}=1 \Longrightarrow \frac{\partial \omega_{i, \mathrm{eq}}^{2}}{\partial \eta_{j}(t)}=\boldsymbol{\phi}_{i, \mathrm{eq}}^{\top} \frac{\partial \boldsymbol{K}_{\mathrm{eq}}}{\partial \eta_{j}(t)} \boldsymbol{\phi}_{i, \mathrm{eq}}$

- Finite difference scheme for tangential stiffness matrix:

$>$ Forward difference: $\left.\quad \frac{\partial \boldsymbol{K}(\boldsymbol{q})}{\partial \eta_{j}(t)}\right|_{\boldsymbol{q}_{\mathrm{eq}}}=\frac{\boldsymbol{K}\left(\boldsymbol{q}_{\mathrm{eq}}+\boldsymbol{\phi}_{j, \mathrm{eq}} \cdot h\right)-\boldsymbol{K}\left(\boldsymbol{q}_{\mathrm{eq}}\right)}{h}$

$>$ Backward difference: $\left.\quad \frac{\partial \boldsymbol{K}(\boldsymbol{q})}{\partial \eta_{j}(t)}\right|_{\boldsymbol{q}_{\mathrm{eq}}}=\frac{\boldsymbol{K}\left(\boldsymbol{q}_{\mathrm{eq}}\right)-\boldsymbol{K}\left(\boldsymbol{q}_{\mathrm{eq}}-\phi_{j, \mathrm{eq}} \cdot h\right)}{h}$

$>$ Central difference: $\left.\quad \frac{\partial \boldsymbol{K}(\boldsymbol{q})}{\partial \eta_{j}(t)}\right|_{\boldsymbol{q}_{\mathrm{eq}}}=\frac{\boldsymbol{K}\left(\boldsymbol{q}_{\mathrm{eq}}+\boldsymbol{\phi}_{j, \mathrm{eq}} \cdot h\right)-\boldsymbol{K}\left(\boldsymbol{q}_{\mathrm{eq}}-\boldsymbol{\phi}_{j, \mathrm{eq}} \cdot h\right)}{2 h}$ 


\section{Modal derivatives / Static modal derivatives}

\section{Handling the singular left-hand side (Ihs)}

By imposing an additional condition/constraint: $\frac{\partial}{\partial \eta_{j}(t)}\left(\phi_{i, \mathrm{eq}}^{\top} \boldsymbol{M} \boldsymbol{\phi}_{i, \mathrm{eq}}\right)=0 \Longrightarrow \boldsymbol{\phi}_{i, \mathrm{eq}}^{\top} \boldsymbol{M} \frac{\partial \phi_{i, \mathrm{eq}}}{\partial \eta_{j}(t)}=0$

- Nelson's method: [Nelson '76]

- Direct method:

$$
\left[\begin{array}{cc}
\left(\boldsymbol{K}_{\mathrm{eq}}-\omega_{i, \mathrm{eq}}^{2} \boldsymbol{M}\right) & -\boldsymbol{M} \boldsymbol{\phi}_{i, \mathrm{eq}} \\
-\left(\boldsymbol{M} \boldsymbol{\phi}_{i, \mathrm{eq}}\right)^{\top} & 0
\end{array}\right]\left[\begin{array}{c}
\boldsymbol{\theta}_{i j} \\
\frac{\partial \omega_{i, \mathrm{eq}}^{2}}{\partial \eta_{j}(t)}
\end{array}\right]=\left[\begin{array}{c}
-\frac{\partial \boldsymbol{K}_{\mathrm{eq}}}{\partial \eta_{j}(t)} \boldsymbol{\phi}_{i, \mathrm{eq}} \\
0
\end{array}\right] \quad \begin{aligned}
& i=1, \ldots, r \\
& j=1, \ldots, r
\end{aligned}
$$

\section{Excluding mass consideration}

Mass terms are usually neglected, leading to the so-called static modal derivatives (SMDs)

$$
\begin{array}{rl}
\left(\boldsymbol{K}_{\mathrm{eq}}-\omega_{i, \mathrm{eq}}^{2} / \boldsymbol{M}\right) \frac{\partial \boldsymbol{\phi}_{i, \mathrm{eq}}}{\partial \eta_{j}(t)}=\left(\frac{\partial \omega_{i, \mathrm{eq}}^{2}}{\partial \eta_{j}(t)} \boldsymbol{M}-\frac{\partial \boldsymbol{K}_{\mathrm{eq}}}{\partial \eta_{j}(t)}\right) \phi_{i, \mathrm{eq}} & \\
\left.\square \quad \boldsymbol{K}_{\mathrm{eq}} \frac{\partial \phi_{i, \mathrm{eq}}}{\partial \eta_{j}(t)}\right|_{\mathrm{s}}=-\frac{\partial \boldsymbol{K}_{\mathrm{eq}}}{\partial \eta_{j}(t)} \phi_{i, \mathrm{eq}} & i=1, \ldots, r \\
& j=1, \ldots, r
\end{array}
$$

Properties:

- Only one factorization of $\boldsymbol{K}_{\text {eq }}$ is needed.

- Static modal derivatives are symmetric: $\boldsymbol{\theta}_{\mathrm{s}, i j}=\left.\frac{\partial \phi_{i, \mathrm{eq}}}{\partial \eta_{j}(t)}\right|_{\mathrm{s}}=\left.\frac{\partial \phi_{j, \mathrm{eq}}}{\partial \eta_{i}(t)}\right|_{\mathrm{s}}=\boldsymbol{\theta}_{\mathrm{s}, j i}$. 


\section{Polynomial system representation}

Nonlinear second-order model

$$
\begin{aligned}
\boldsymbol{M} \ddot{\boldsymbol{q}}(t)+\boldsymbol{D} \dot{\boldsymbol{q}}(t)+\boldsymbol{f}(\boldsymbol{q}(t)) & =\boldsymbol{B} \boldsymbol{F}(t) \quad \boldsymbol{q}(0)=\boldsymbol{q}_{0}, \dot{\boldsymbol{q}}(0)=\dot{\boldsymbol{q}}_{0} \\
\boldsymbol{y}(t) & =\boldsymbol{C} \boldsymbol{q}(t)
\end{aligned}
$$

Taylor series expansion

$$
\begin{aligned}
& \boldsymbol{f}(\boldsymbol{q})=\boldsymbol{f}\left(\boldsymbol{q}_{\mathrm{eq}}\right)+\frac{\partial \boldsymbol{f}\left(\boldsymbol{q}_{\mathrm{eq}}\right)}{\partial \boldsymbol{q}}\left(\boldsymbol{q}-\boldsymbol{q}_{\mathrm{eq}}\right)+\frac{1}{2 !} \frac{\partial^{2} \boldsymbol{f}\left(\boldsymbol{q}_{\mathrm{eq}}\right)}{\partial \boldsymbol{q}^{2}}\left(\boldsymbol{q}-\boldsymbol{q}_{\mathrm{eq}}\right)^{(2)}+\frac{1}{3 !} \frac{\partial^{3} \boldsymbol{f}\left(\boldsymbol{q}_{\mathrm{eq}}\right)}{\partial \boldsymbol{q}^{3}}\left(\boldsymbol{q}-\boldsymbol{q}_{\mathrm{eq}}\right)^{(3)}+\cdots \\
& \boldsymbol{q}_{\mathrm{eq}}=\mathbf{0}
\end{aligned}
$$

Polynomial (cubic) nonlinear second-order model

$$
\boldsymbol{M} \ddot{\boldsymbol{q}}(t)+\boldsymbol{D} \dot{\boldsymbol{q}}(t)+\boldsymbol{K}^{(1)} \boldsymbol{q}(t)+\boldsymbol{K}^{(2)}(\boldsymbol{q}(t) \otimes \boldsymbol{q}(t))+\boldsymbol{K}^{(3)}(\boldsymbol{q}(t) \otimes \boldsymbol{q}(t) \otimes \boldsymbol{q}(t))=\boldsymbol{B} \boldsymbol{F}(t)
$$

Symmetric tensors $\boldsymbol{K}^{(1)} \in \mathbb{R}^{n \times n}, \mathcal{K}^{(2)} \in \mathbb{R}^{n \times n \times n}$ and $\mathcal{K}^{(3)} \in \mathbb{R}^{n \times n \times n \times n}$

$$
K_{a b}^{(1)}=\frac{\partial f_{a}}{\partial q_{b}}=\frac{\partial^{2} \mathcal{V}}{\partial q_{a} \partial q_{b}}, \quad \mathcal{K}_{a b c}^{(2)}=\frac{1}{2} \frac{\partial^{2} f_{a}}{\partial q_{b} \partial q_{c}}=\frac{1}{2} \frac{\partial^{3} \mathcal{V}}{\partial q_{a} \partial q_{b} \partial q_{c}}, \quad \mathcal{K}_{a b c d}^{(3)}=\frac{1}{6} \frac{\partial^{3} f_{a}}{\partial q_{b} \partial q_{c} \partial q_{d}}=\frac{1}{6} \frac{\partial^{4} \mathcal{V}}{\partial q_{a} \partial q_{b} \partial q_{c} \partial q_{d}}
$$




\section{Volterra theory and variational equations}

For an input of the form $\alpha \boldsymbol{F}(t)$, it is assumed that the response is

$$
\begin{aligned}
& \boldsymbol{q}(t)=\alpha \boldsymbol{q}_{1}(t)+\alpha^{2} \boldsymbol{q}_{2}(t)+\ldots, \\
& \ddot{\boldsymbol{q}}(t)=\alpha \ddot{\boldsymbol{q}}_{1}(t)+\alpha^{2} \ddot{\boldsymbol{q}}_{2}(t)+\ldots
\end{aligned}
$$

Inserting the assumed input and the assumed response into the polynomial system, yields

$$
\begin{aligned}
& \boldsymbol{M}\left(\alpha \ddot{\boldsymbol{q}}_{1}(t)+\alpha^{2} \ddot{\boldsymbol{q}}_{2}(t)+\ldots\right)+\boldsymbol{K}^{(1)}\left(\alpha \boldsymbol{q}_{1}(t)+\alpha^{2} \boldsymbol{q}_{2}(t)+\ldots\right) \\
& +\boldsymbol{K}^{(2)}(\boldsymbol{q}(t) \otimes \boldsymbol{q}(t))+\boldsymbol{K}^{(3)}(\boldsymbol{q}(t) \otimes \boldsymbol{q}(t) \otimes \boldsymbol{q}(t))=\boldsymbol{B} \alpha \boldsymbol{F}(t) .
\end{aligned}
$$

Equating coefficients of $\alpha^{k}$, yields the variational equations $(\boldsymbol{D}=\mathbf{0})$

$$
\begin{array}{clc}
\alpha: & \boldsymbol{M} \ddot{\boldsymbol{q}}_{1}(t)+\boldsymbol{K}^{(1)} \boldsymbol{q}_{1}(t)=\boldsymbol{B} \boldsymbol{F}(t), & \boldsymbol{q}_{1}(0)=\boldsymbol{q}_{0}, \\
\alpha^{2}: & \boldsymbol{M} \ddot{\boldsymbol{q}}_{2}(t)+\boldsymbol{K}^{(1)} \boldsymbol{q}_{2}(t)=-\boldsymbol{K}^{(2)}\left(\boldsymbol{q}_{1}(t) \otimes \boldsymbol{q}_{1}(t)\right), & \boldsymbol{q}_{2}(0)=\mathbf{0}, \\
\alpha^{3}: & \boldsymbol{M} \ddot{\boldsymbol{q}}_{3}(t)+\boldsymbol{K}^{(1)} \boldsymbol{q}_{3}(t) & \boldsymbol{q}_{3}(0)=\mathbf{0}, \\
& \quad=-\boldsymbol{K}^{(2)}\left(\boldsymbol{q}_{1}(t) \otimes \boldsymbol{q}_{2}(t)+\boldsymbol{q}_{2}(t) \otimes \boldsymbol{q}_{1}(t)\right)-\boldsymbol{K}^{(3)}\left(\boldsymbol{q}_{1}(t) \otimes \boldsymbol{q}_{1}(t) \otimes \boldsymbol{q}_{1}(t)\right)
\end{array}
$$

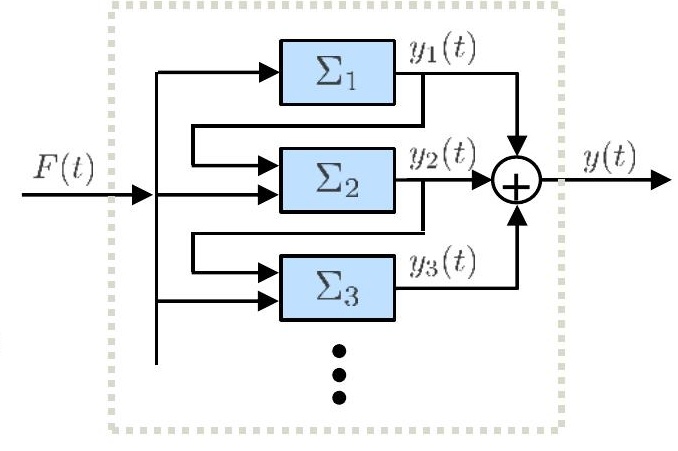

series of cascaded subsystems 


\section{Novel derivation of modal derivatives}

\section{Modes (First subsystem)}

Subsystem state-equation: $\quad \boldsymbol{M} \ddot{\boldsymbol{q}}_{1}(t)+\boldsymbol{K}^{(1)} \boldsymbol{q}_{1}(t)=\boldsymbol{B} \boldsymbol{F}(t)$

Ansatz for the homogeneous solution: $\boldsymbol{q}_{1}(t)=\sum_{i=1}^{n} c_{i} \phi_{i} \cos \left(\omega_{i} t\right)$

Inserting ansatz (with $\left.\ddot{\boldsymbol{q}}_{1}(t)\right)$ in state-equation yields: $\left(\boldsymbol{K}^{(1)}-\omega_{i}^{2} \boldsymbol{M}\right) \boldsymbol{\phi}_{i} \sum_{i=1}^{n} c_{i} \cos \left(\omega_{i} t\right)=\mathbf{0}$,

\section{Modal derivatives (Second subsystem)}

Subsystem state-equation: $\quad \boldsymbol{M} \ddot{\boldsymbol{q}}_{2}(t)+\boldsymbol{K}^{(1)} \boldsymbol{q}_{2}(t)=-\boldsymbol{K}^{(2)}\left(\boldsymbol{q}_{1}(t) \otimes \boldsymbol{q}_{1}(t)\right)$

$\begin{gathered}\text { Ansatz for the particular solution: } \\ \text { (method of undetermined coeff.) }\end{gathered} \boldsymbol{q}_{2}(t)=\sum_{i=1}^{n} \sum_{j=1}^{n} \frac{1}{2} c_{i} c_{j}\left(\widetilde{\boldsymbol{\theta}}_{i j} \cos \left(\left(\omega_{i}+\omega_{j}\right) t\right)+\widetilde{\widetilde{\boldsymbol{\theta}}}_{i j} \cos \left(\left(\omega_{i}-\omega_{j}\right) t\right)\right)$

Inserting ansatz (with $\ddot{\boldsymbol{q}}_{2}(t)$ and $\boldsymbol{q}_{1}(t) \otimes \boldsymbol{q}_{1}(t)$ ) in state-equation yields exemplarily:

$$
\begin{aligned}
\mathbf{0} & =\frac{1}{2} c_{1}^{2} \cos \left(2 \omega_{1} t\right) \underbrace{\left(\left(-\left(2 \omega_{1}\right)^{2} \boldsymbol{M}+\boldsymbol{K}^{(1)}\right) \tilde{\boldsymbol{\theta}}_{11}+\boldsymbol{K}^{(2)}\left(\boldsymbol{\phi}_{1} \otimes \boldsymbol{\phi}_{1}\right)\right)}_{=\mathbf{0}}+\frac{1}{2} c_{1}^{2} \underbrace{\left(\boldsymbol{K}^{(1)} \widetilde{\tilde{\boldsymbol{\theta}}}_{11}+\boldsymbol{K}^{(2)}\left(\boldsymbol{\phi}_{1} \otimes \boldsymbol{\phi}_{1}\right)\right)}_{=\mathbf{0}}+\cdots \\
& +\frac{1}{2} c_{1} c_{2} \cos \left(\left(\omega_{1}+\omega_{2}\right) t\right) \underbrace{\left(\left(-\left(\omega_{1}+\omega_{2}\right)^{2} \boldsymbol{M}+\boldsymbol{K}^{(1)}\right)\left(\widetilde{\boldsymbol{\theta}}_{12}+\widetilde{\boldsymbol{\theta}}_{21}\right) \boldsymbol{K}^{(2)}\left(\boldsymbol{\phi}_{1} \otimes \boldsymbol{\phi}_{2}+\boldsymbol{\phi}_{2} \otimes \boldsymbol{\phi}_{1}\right)\right)}_{=\mathbf{0}}+\cdots
\end{aligned}
$$




\section{New modal derivatives}

It follows from all brackets:

$$
\begin{array}{ll}
\left(\boldsymbol{K}^{(1)}-\left(\omega_{i}+\omega_{j}\right)^{2} \boldsymbol{M}\right) \widetilde{\boldsymbol{\theta}}_{i j}=-\boldsymbol{K}^{(2)}\left(\boldsymbol{\phi}_{i} \otimes \boldsymbol{\phi}_{j}\right), & i, j=1, \ldots, r, \\
\left(\boldsymbol{K}^{(1)}-\left(\omega_{i}-\omega_{j}\right)^{2} \boldsymbol{M}\right) \widetilde{\widetilde{\boldsymbol{\theta}}}_{i j}=-\boldsymbol{K}^{(2)}\left(\boldsymbol{\phi}_{i} \otimes \boldsymbol{\phi}_{j}\right), \quad i, j=1, \ldots, r .
\end{array}
$$

Equivalent description for the right-hand side

$$
\begin{gathered}
\frac{\partial K_{a b}^{(1)}}{\partial \eta_{j}(t)}\left(\boldsymbol{\phi}_{i}\right)_{b}=\frac{\partial K_{a b}^{(1)}}{\partial q_{c}} \frac{\partial q_{c}}{\partial \eta_{j}(t)}\left(\boldsymbol{\phi}_{i}\right)_{b}:=\frac{\partial^{2} f_{a}}{\partial q_{b} \partial q_{c}}\left(\boldsymbol{\phi}_{j}\right)_{c}\left(\boldsymbol{\phi}_{i}\right)_{b}=2 \mathcal{K}_{a b c}^{(2)}\left(\boldsymbol{\phi}_{j}\right)_{c}\left(\boldsymbol{\phi}_{i}\right)_{b} . \\
\left.\frac{\partial \boldsymbol{K}^{(1)}(\boldsymbol{q})}{\partial \eta_{j}(t)}\right|_{\boldsymbol{q}_{\mathrm{eq}}} \boldsymbol{\phi}_{i}:=2 \boldsymbol{K}^{(2)}\left(\boldsymbol{\phi}_{i} \otimes \boldsymbol{\phi}_{j}\right)=\boldsymbol{K}^{(2)}\left(\boldsymbol{\phi}_{i} \otimes \boldsymbol{\phi}_{j}+\boldsymbol{\phi}_{j} \otimes \boldsymbol{\phi}_{i}\right) .
\end{gathered}
$$

Thus, the new modal derivatives are given by:

$$
\begin{aligned}
& \left(\boldsymbol{K}^{(1)}-\left(\omega_{i}+\omega_{j}\right)^{2} \boldsymbol{M}\right) \widetilde{\boldsymbol{\theta}}_{i j}=-\left.\frac{1}{2} \frac{\partial \boldsymbol{K}^{(1)}(\boldsymbol{q})}{\partial \eta_{j}(t)}\right|_{\boldsymbol{q}_{\mathrm{eq}}} \boldsymbol{\phi}_{i}, \quad i, j=1, \ldots, r, \\
& \left(\boldsymbol{K}^{(1)}-\left(\omega_{i}-\omega_{j}\right)^{2} \boldsymbol{M}\right) \widetilde{\widetilde{\boldsymbol{\theta}}}_{i j}=-\left.\frac{1}{2} \frac{\partial \boldsymbol{K}^{(1)}(\boldsymbol{q})}{\partial \eta_{j}(t)}\right|_{\boldsymbol{q}_{\mathrm{eq}}} \boldsymbol{\phi}_{i}, \quad i, j=1, \ldots, r .
\end{aligned}
$$




\section{Comments on the gained new derivatives}

\section{Conventional modal derivatives}

$$
\begin{aligned}
\left(\boldsymbol{K}_{\mathrm{eq}}-\omega_{i, \mathrm{eq}}^{2} \boldsymbol{M}\right) \boldsymbol{\theta}_{i j} & =\left(\frac{\partial \omega_{i, \mathrm{eq}}^{2}}{\partial \eta_{j}(t)} \boldsymbol{M}-\frac{\partial \boldsymbol{K}_{\mathrm{eq}}}{\partial \eta_{j}(t)}\right) \boldsymbol{\phi}_{i, \mathrm{eq}} \\
\boldsymbol{K}_{\mathrm{eq}} \boldsymbol{\theta}_{\mathrm{s}, i j} & =-\frac{\partial \boldsymbol{K}_{\mathrm{eq}}}{\partial \eta_{j}(t)} \boldsymbol{\phi}_{i, \mathrm{eq}}
\end{aligned}
$$

\section{Properties:}

- Single eigenfrequency

- Rhs of MDs and SMDs are different

- Neglection of mass $\rightarrow$ SMDs

- SMDs only obtained when neglecting mass

- MDs are (in general) NOT symmetric

- Singular linear system of equations

\section{Volterra series-based modal derivatives}

$$
\begin{aligned}
& \left(\boldsymbol{K}^{(1)}-\left(\omega_{i}+\omega_{j}\right)^{2} \boldsymbol{M}\right) \widetilde{\boldsymbol{\theta}}_{i j}=-\left.\frac{1}{2} \frac{\partial \boldsymbol{K}^{(1)}(\boldsymbol{q})}{\partial \eta_{j}(t)}\right|_{\boldsymbol{q}_{\mathrm{eq}}} \boldsymbol{\phi}_{i}, \\
& \left(\boldsymbol{K}^{(1)}-\left(\omega_{i}-\omega_{j}\right)^{2} \boldsymbol{M}\right) \widetilde{\widetilde{\boldsymbol{\theta}}}_{i j}=-\left.\frac{1}{2} \frac{\partial \boldsymbol{K}^{(1)}(\boldsymbol{q})}{\partial \eta_{j}(t)}\right|_{\boldsymbol{q}_{\mathrm{eq}}} \boldsymbol{\phi}_{i},
\end{aligned}
$$

\section{Properties:}

- Sum/subtraction of eigenfrequencies

- Rhs for new MDs is the same as for SMDs!

- Neglection of mass $\rightarrow \widetilde{\boldsymbol{\theta}}_{i j}=\widetilde{\widetilde{\boldsymbol{\theta}}}_{i j}=\boldsymbol{\theta}_{\mathrm{s}, i j}$

- Cancelation of eigenfrequencies: $\widetilde{\widetilde{\theta}}_{i i}=\boldsymbol{\theta}_{\mathrm{s}, i j}$

- New MDs are symmetric!

- Regular linear system of equations

$>$ only singular, if sum/subtraction of eigenfrequencies is again eigenfrequency 


\section{Possible applications of the new derivatives}

\section{1.) Assess approximation quality of Volterra approximation}

Compare analytical solution given by the first and second subsystem

$$
\boldsymbol{q}_{1 . .2}(t)=\underbrace{\sum_{i=1}^{n} c_{i} \boldsymbol{\phi}_{i} \cos \left(\omega_{i} t\right)}_{\boldsymbol{q}_{1}(t)}+\underbrace{\sum_{i=1}^{n} \sum_{j=1}^{n} \frac{1}{2} c_{i} c_{j}\left(\widetilde{\boldsymbol{\theta}}_{i j} \cos \left(\left(\omega_{i}+\omega_{j}\right) t\right)+\widetilde{\boldsymbol{\theta}}_{i j} \cos \left(\left(\omega_{i}-\omega_{j}\right) t\right)\right)}_{\boldsymbol{q}_{2}(t)}
$$

with simulated solution $\boldsymbol{q}_{\text {sim }}(t)$ or solution given by superposing nonlinear normal modes

Reformulated analytical solution using trigonometric identities

$$
\begin{array}{rlr}
\boldsymbol{q}_{2}(t) & =\sum_{i=1}^{n} \sum_{j=1}^{n} \frac{1}{2} c_{i} c_{j}\left(\widetilde{\boldsymbol{\theta}}_{i j} \cos \left(\left(\omega_{i}+\omega_{j}\right) t\right)+\widetilde{\widetilde{\boldsymbol{\theta}}}_{i j} \cos \left(\left(\omega_{i}-\omega_{j}\right) t\right)\right) & \overline{\boldsymbol{\theta}}_{i j}=\frac{1}{2}\left(\widetilde{\boldsymbol{\theta}}_{i j}+\widetilde{\boldsymbol{\theta}}_{i j}\right) \\
& =\sum_{i=1}^{n} \sum_{j=1}^{n} c_{i} c_{j}\left(\overline{\boldsymbol{\theta}}_{i j} \cos \left(\omega_{i} t\right) \cos \left(\omega_{j} t\right)-\widehat{\boldsymbol{\theta}}_{i j} \sin \left(\omega_{i} t\right) \sin \left(\omega_{j} t\right)\right) & \widehat{\boldsymbol{\theta}}_{i j}=\frac{1}{2}\left(\widetilde{\boldsymbol{\theta}}_{i j}-\widetilde{\boldsymbol{\theta}}_{i j}\right)
\end{array}
$$$$
\boldsymbol{q}_{1 . .2}(t)=\underbrace{\sum_{i=1}^{n} c_{i} \boldsymbol{\phi}_{i} \cos \left(\omega_{i} t\right)}_{\boldsymbol{q}_{1}(t)}+\underbrace{\sum_{i=1}^{n} \sum_{j=1}^{n} c_{i} c_{j}\left(\overline{\boldsymbol{\theta}}_{i j} \cos \left(\omega_{i} t\right) \cos \left(\omega_{j} t\right)-\widehat{\boldsymbol{\theta}}_{i j} \sin \left(\omega_{i} t\right) \sin \left(\omega_{j} t\right)\right)}_{\boldsymbol{q}_{2}(t)}
$$ 


\section{Possible applications of the new derivatives}

2.) Novel quadratic manifold approaches for model order reduction

- Common quadratic manifold approach

$$
\begin{aligned}
\boldsymbol{q}(t) & \approx \sum_{i=1}^{r} \boldsymbol{\phi}_{i} q_{\mathrm{r}, i}(t)+\sum_{i=1}^{r} \sum_{j=1}^{r} \boldsymbol{\theta}_{i j} q_{\mathrm{r}, i}(t) q_{\mathrm{r}, j}(t) \\
& =\boldsymbol{\Phi}_{r} \boldsymbol{q}_{\mathrm{r}}(t)+\boldsymbol{\Theta}_{r^{2}}\left(\boldsymbol{q}_{\mathrm{r}}(t) \otimes \boldsymbol{q}_{\mathrm{r}}(t)\right)
\end{aligned}
$$

Using symmetrized MDs $\boldsymbol{\theta}_{i j}$ or static MDs (SMDs) $\boldsymbol{\theta}_{\mathrm{s}, i j}$

- Novel quadratic manifold approach (1)

$$
\begin{aligned}
\boldsymbol{q}(t) & \approx \sum_{i=1}^{r} \boldsymbol{\phi}_{i} q_{\mathrm{r}, i}(t)+\sum_{i=1}^{r} \sum_{j=1}^{r} \overline{\boldsymbol{\theta}}_{i j} q_{\mathrm{r}, i}(t) q_{\mathrm{r}, j}(t) \\
& =\boldsymbol{\Phi}_{r} \boldsymbol{q}_{\mathrm{r}}(t)+\overline{\boldsymbol{\Theta}}_{r^{2}}\left(\boldsymbol{q}_{\mathrm{r}}(t) \otimes \boldsymbol{q}_{\mathrm{r}}(t)\right)
\end{aligned}
$$

- Novel quadratic manifold approach (2)

$$
\begin{aligned}
\boldsymbol{q}(t) & \approx \sum_{i=1}^{r} \boldsymbol{\phi}_{i} q_{\mathrm{r}, i}(t)+\sum_{i=1}^{r} \sum_{j=1}^{r} \overline{\boldsymbol{\theta}}_{i j} q_{\mathrm{r}, i}(t) q_{\mathrm{r}, j}(t)-\frac{1}{\omega_{i} \omega_{j}} \widehat{\boldsymbol{\theta}}_{i j} \dot{\mathrm{q}}_{\mathrm{r}, i}(t) \dot{q}_{\mathrm{r}, j}(t) \\
& =\boldsymbol{\Phi}_{r} \boldsymbol{q}_{\mathrm{r}}(t)+\overline{\boldsymbol{\Theta}}_{r^{2}}\left(\boldsymbol{q}_{\mathrm{r}}(t) \otimes \boldsymbol{q}_{\mathrm{r}}(t)\right)-\widehat{\boldsymbol{\Theta}}_{r^{2}}\left(\dot{\boldsymbol{q}}_{\mathrm{r}}(t) \otimes \dot{\boldsymbol{q}}_{\mathrm{r}}(t)\right)
\end{aligned}
$$

\section{Reduced coordinates:}

$$
\begin{aligned}
& q_{\mathrm{r}, i}(t)=c_{i} \cos \left(\omega_{i} t\right), \\
& q_{\mathrm{r}, i}(t) q_{\mathrm{r}, j}(t)=c_{i} c_{j} \cos \left(\omega_{i} t\right) \cos \left(\omega_{j} t\right) \\
& \begin{array}{l}
\overline{\boldsymbol{\theta}}_{i j}=\frac{1}{2}\left(\widetilde{\boldsymbol{\theta}}_{i j}+\widetilde{\widetilde{\boldsymbol{\theta}}}_{i j}\right) \\
\widehat{\boldsymbol{\theta}}_{i j}=\frac{1}{2}\left(\widetilde{\boldsymbol{\theta}}_{i j}-\widetilde{\widetilde{\boldsymbol{\theta}}}_{i j}\right)
\end{array}
\end{aligned}
$$

Reduced velocities:

$\dot{q}_{\mathrm{r}, i}(t) \dot{q}_{\mathrm{r}, j}(t)=c_{i} c_{j} \omega_{i} \omega_{j} \sin \left(\omega_{i} t\right) \sin \left(\omega_{j} t\right)$ 


\section{Possible applications of the new derivatives}

Dimensional reduction - Roadmap and Workflow

$$
\begin{gathered}
\text { Basis augmentation } \\
\boldsymbol{q}(t) \approx \boldsymbol{V}_{\text {aug }} \boldsymbol{q}_{\mathrm{r}, \text { aug }}(t) \\
\boldsymbol{V}_{\text {aug }}=\left[\boldsymbol{\Phi}_{r}, \boldsymbol{\Theta}_{r^{2}}\right], \quad \boldsymbol{V}_{\text {aug }}=\left[\boldsymbol{\Phi}_{r}, \boldsymbol{\Theta}_{\mathrm{s}}\right] \\
\boldsymbol{V}_{\text {aug }}=\left[\boldsymbol{\Phi}_{r}, \widetilde{\boldsymbol{\Theta}}, \widetilde{\boldsymbol{\Theta}}\right], \quad \boldsymbol{V}_{\text {aug }}=\left[\boldsymbol{\Phi}_{r}, \overline{\boldsymbol{\Theta}}, \widehat{\boldsymbol{\Theta}}\right]
\end{gathered}
$$

Application to nonlinear system

$$
\begin{aligned}
M \ddot{q}+f(q) & =B F \\
y & =C q
\end{aligned}
$$

\section{Evaluation in time-domain}

- Compare FOM and ROM via simulation runs for different inputs

- Compare ROMs with POD-ROM

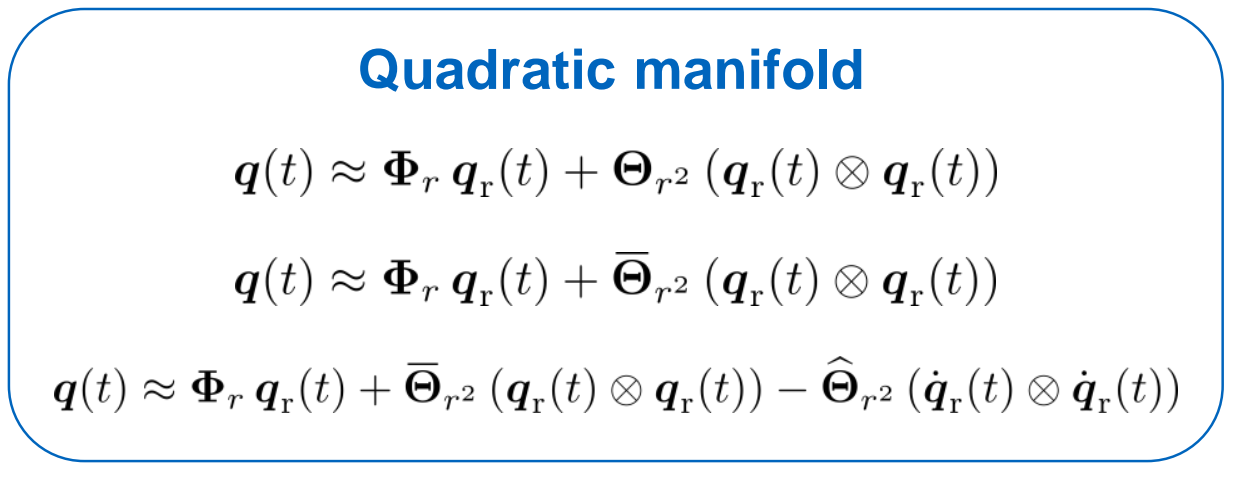

Application to polynomial system

$$
\begin{aligned}
\boldsymbol{M} \ddot{\boldsymbol{q}}+\boldsymbol{K}^{(1)} \boldsymbol{q}+\boldsymbol{K}^{(2)}(\boldsymbol{q} \otimes \boldsymbol{q})+\boldsymbol{K}^{(3)}(\boldsymbol{q} \otimes \boldsymbol{q} \otimes \boldsymbol{q}) & =\boldsymbol{B} \boldsymbol{F} \\
\boldsymbol{y} & =\boldsymbol{C} \boldsymbol{q}
\end{aligned}
$$

\section{Evaluation in frequency-domain}

- Compute NNMs and NLFRF via shooting and path continuation

- Compare NNMs and NLFRFs of FOM and differently obtained ROMs 


\section{Preliminary simulation - Cantilever Beam}

2D model of a cantilever beam

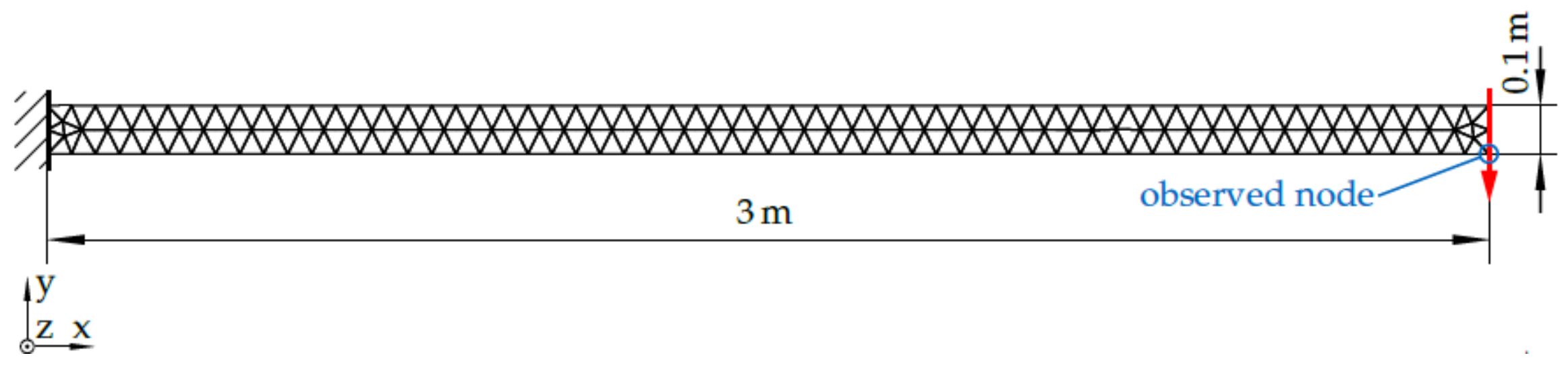

- 246 triangular Tri6 elements; 1224 dofs

- linear St. Venant-Kirchhoff material

- geometric nonlinear behaviour

- loading force at the tip in negative y-direction

- simulation conducted with open-source AMfe-code

- reduction via basis augmentation with new MDs 


\section{Preliminary simulation - Cantilever Beam}

Displacement of front tip over time

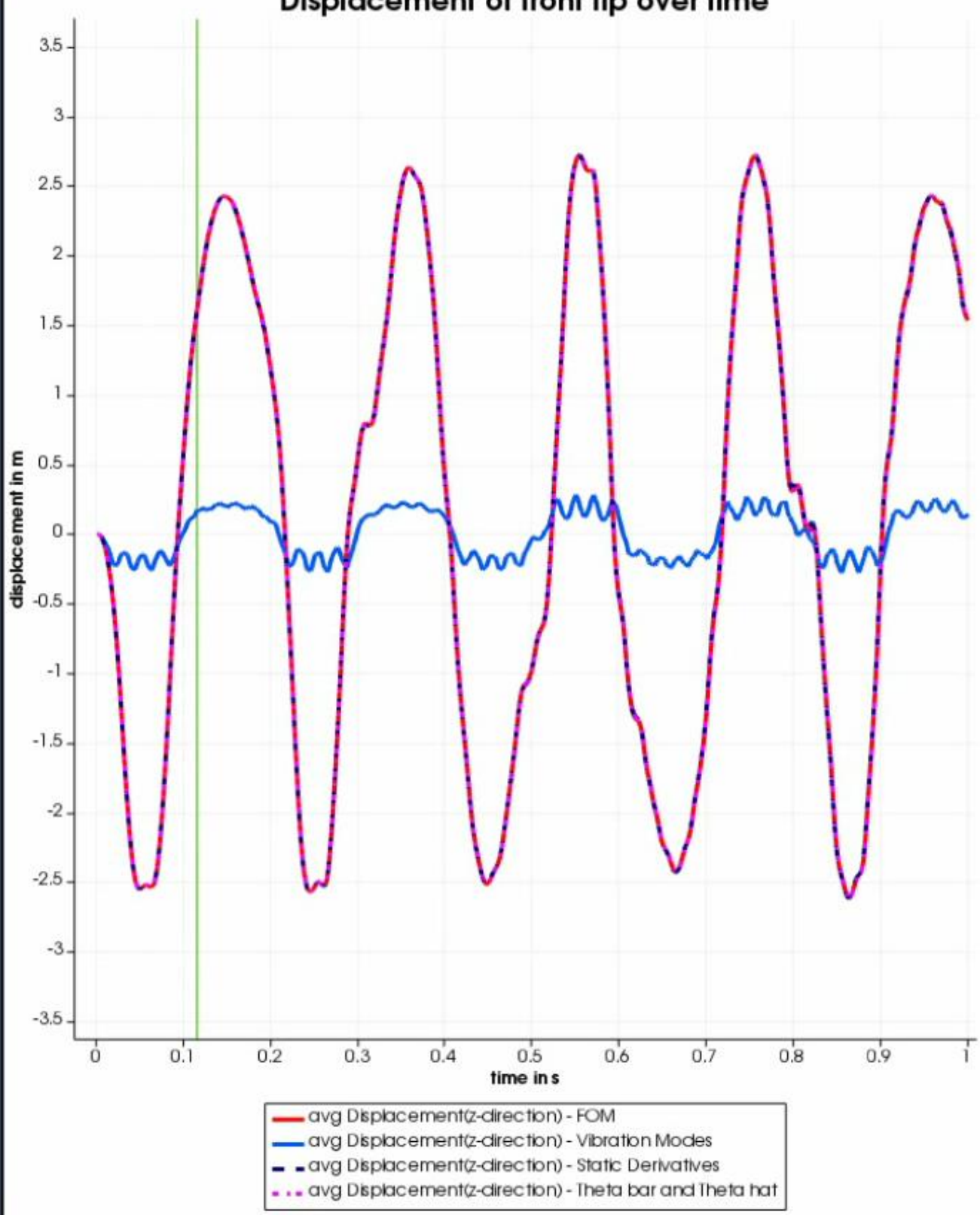




\section{Summary \& Outlook}

\section{Take-Home Messages:}

- Model reduction with modal derivatives to capture (geometric) nonlinear behaviour

- Classical derivation of MDs is based on perturbation of the linearized eigenvalue problem

- Novel derivation based on Volterra series yields slightly different expressions for MDs

- Novel MDs are inherently symmetric; static derivatives can be retrieved from the new MDs

- Possible promising applications in nonlinear model order reduction

\section{Ongoing / Future Work:}

- Implementation of novel quadratic manifold approaches

- Validation of ROMs in time-, but also in frequency-domain (NLFRFs)

- Hyper-Reduction

\section{Thank you for your attention!}

